\title{
Supplement to Bayesian Indirect Inference using a Parametric Auxiliary Model
}

Christopher C. Drovandi Anthony N. Pettitt and Anthony Lee

\section{APPENDIX A}

We let

$$
p(y \mid \theta)=\mathcal{N}(y ; \theta, 1),
$$

and so the $\mathrm{ABC}$ artificial likelihood, with $K_{\epsilon}\left(\rho\left(s\left(x_{1: n}\right), s(y)\right)\right)=\mathcal{N}\left(\rho\left(s\left(x_{1: n}\right), s(y)\right) ; 0, \epsilon\right)$ and $s\left(x_{1: n}\right)=\frac{1}{n} \sum_{j=1}^{n} x_{i}$ is

$$
p_{\epsilon, n}(y \mid \theta)=\mathcal{N}\left(y ; \theta, n^{-1}+\epsilon\right)
$$

and if the prior on $\theta$ is $p(\theta)=\mathcal{N}\left(\theta ; 0, \sigma^{2}\right)$ then the posterior is

$$
\begin{aligned}
p_{\epsilon, n}(\theta \mid y) & =\mathcal{N}\left(\theta ; \frac{y}{\left(n^{-1}+\epsilon\right)} \cdot\left[\frac{1}{n^{-1}+\epsilon}+\frac{1}{\sigma^{2}}\right]^{-1},\left[\frac{1}{n^{-1}+\epsilon}+\frac{1}{\sigma^{2}}\right]^{-1}\right) \\
& =\mathcal{N}\left(\theta ; \frac{y \sigma^{2}}{\sigma^{2}+n^{-1}+\epsilon}, \frac{\sigma^{2}\left(n^{-1}+\epsilon\right)}{\sigma^{2}+n^{-1}+\epsilon}\right)
\end{aligned}
$$

whereas

$$
\begin{aligned}
p(\theta \mid y) & =\mathcal{N}\left(\theta ; y \cdot\left[1+\frac{1}{\sigma^{2}}\right]^{-1},\left[1+\frac{1}{\sigma^{2}}\right]^{-1}\right) \\
& =\mathcal{N}\left(\theta ; \frac{y \sigma^{2}}{\sigma^{2}+1}, \frac{\sigma^{2}}{\sigma^{2}+1}\right) .
\end{aligned}
$$

In this example the $\mathrm{ABC}$ posterior mean moves closer to $y$ and has a variance tending to 0 as $n \rightarrow \infty$ and $\epsilon \rightarrow 0$.

School of Mathematical Sciences, Queensland University of Technology, Brisbane, Australia, 4000 (e-mail: c.drovandi@qut.edu.au; a.pettitt@qut.edu.au) Department of Statistics, University of Warwick, Coventry, UK CV4 7AL (e-mail: anthony.lee@warwick.ac.uk) 


\section{APPENDIX B}

Applying the same process as in the main paper, a four component auxiliary mixture model produced a p-value of roughly 0.26 , indicating a much better fitting auxiliary model. The BIC of the four component mixture model was 32114 compared to 32401 for the three component mixture model. The superior fit of the four component model would indicate that summary statistics drawn from the auxiliary model would carry more of the information of the observed dataset. However, an extra component creates three additional summary statistics, which could create issues for ABC II. Furthermore, the EM algorithm applied to four components was much more expensive and the algorithm was more prone to getting stuck in local modes. In terms of the BIC, it was found that a five component mixture model fits best, but using this was not computationally feasible.

The results comparing the pBII methods when the four component mixture auxiliary model was used is shown in Figure 1. The ABC II methods with regression adjustment produced similar results and outperformed pdBIL. Further, the ABC IS approach was able to avoid the heavy computation associated with fitting the four component mixture model at every iteration and thus also avoids other numerical issues such as the EM algorithm converging to potentially local optima. When comparing Figure 1 of this appendix with Figure 7 of the main paper it is evident that using the four component auxiliary mixture model provides some improvement to the results of $g$ and $k$ for ABC II. This is not surprising given that the summaries obtained from the four component mixture model should carry more of the information contained in the observed data.

Figures 2 and 3 show the results for ABC II when no regression adjustment is applied when using the three and four component auxiliary mixture model, respectively. Here it can be seen that the posterior distributions based on the three component mixture model tend to be more precise than that of the four component mixture model (especially for parameters $a$ and $b$ ). This can be explained by the increase in dimension of the summary statistic for the four component mixture model. However, as has been mentioned, the results for the four com-

imsart-sts ver. 2013/03/06 file: Bayes_Ind_inf_Revision_For_SS_Supplement.tex date: July 15, 2014 


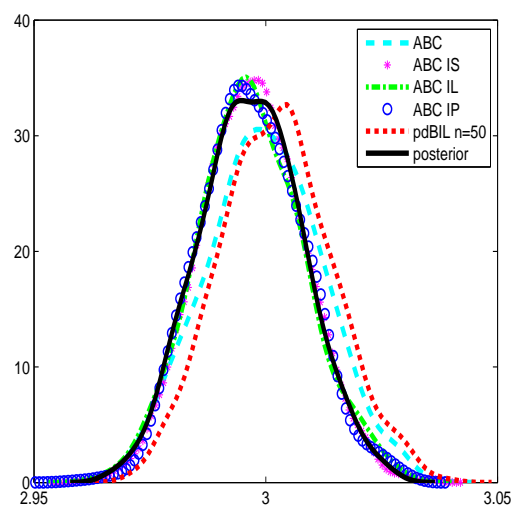

(a) $a$

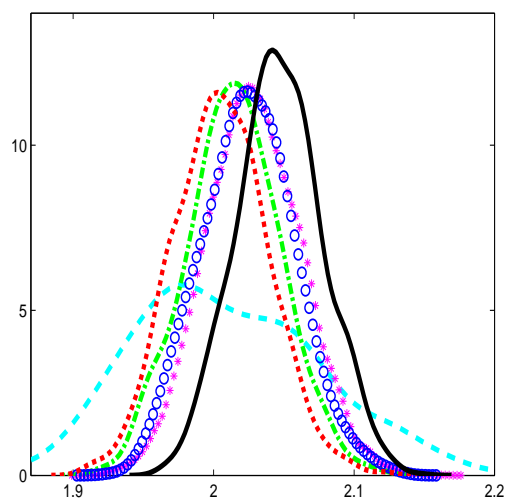

(c) $g$

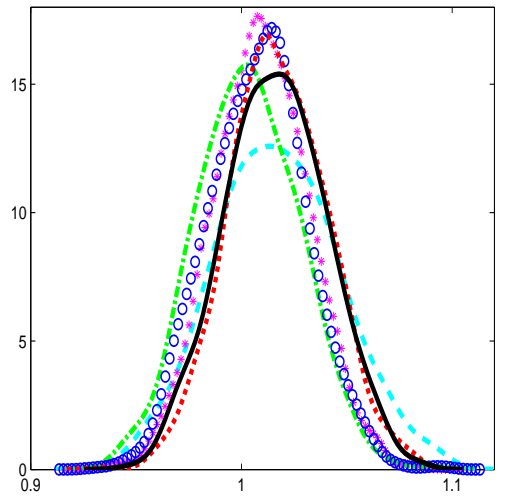

(b) $b$

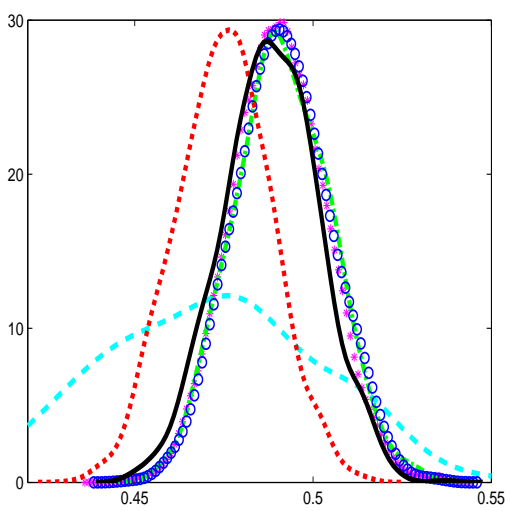

(d) $k$

FIG 1. Posterior distributions for the parameters of the g-and-k model based on the ABC approach of Drovandi and Pettitt (2011) (cyan dash), ABC IS (purple star), ABC IL (green dot-dash), $A B C$ IP (blue circle) and pdBIL with $n=50$ (red dot) and results from using the true likelihood (black solid). Note that regression adjustment has been applied to all ABC results. Here a four component normal mixture model was used as the auxiliary model (on-line figure in colour). 


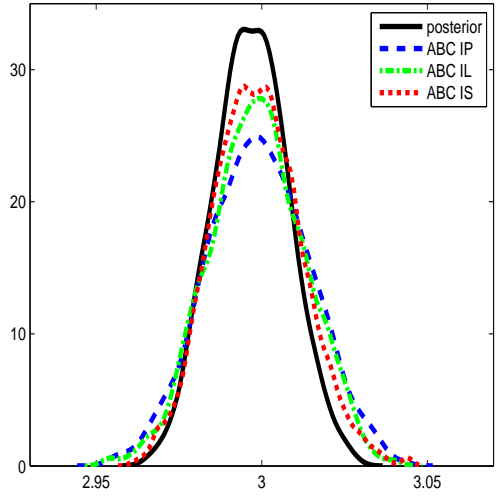

(a) $a$

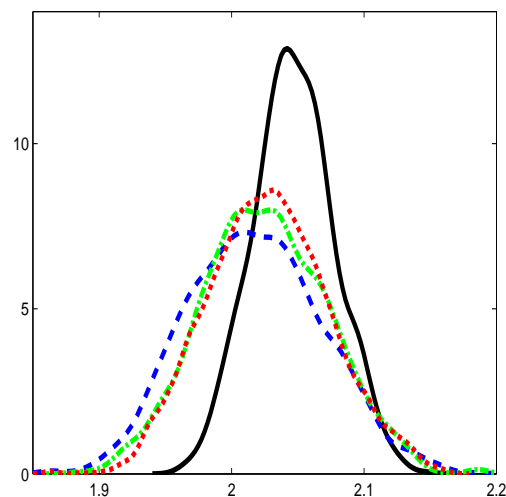

(c) $g$

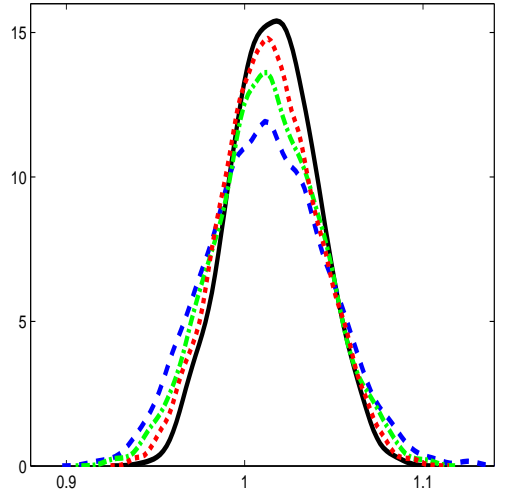

(b) $b$

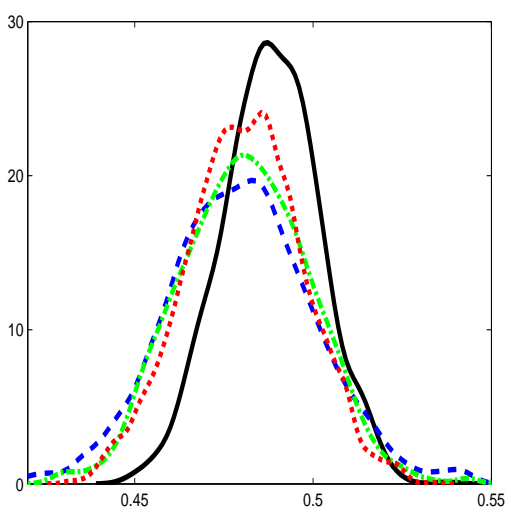

(d) $k$

FIG 2. Posterior distributions for the parameters of the g-and-k model based on the ABC II approaches without applying any regression adjustment. Results are presented for ABC IP (blue dash), $A B C$ IL (green dot-dash), ABC IS (red dot) and from using the true likelihood (black solid). Here a three component normal mixture model was used as the auxiliary model (on-line figure in colour).

ponent mixture model outperform those of the three component mixture model model following regression adjustment. The positive impact of applying regression adjustment in this example can be seen when comparing Figures 1 and 3.

\section{APPENDIX C}

Here we describe the results for the pBII methods when applied to the simulated macroparasite data of the main article. To determine the goodness-of-fit of the auxiliary model (we use the same auxiliary model specified in the main article) to this data, we considered the following based on the generalised Pearson 


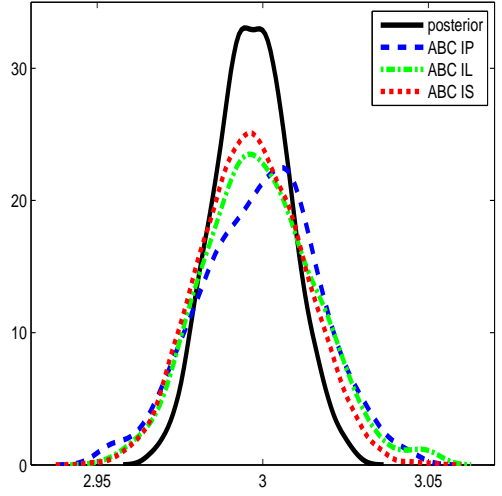

(a) $a$

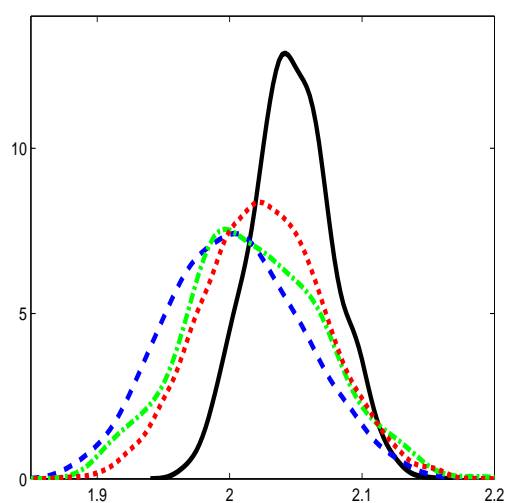

(c) $g$

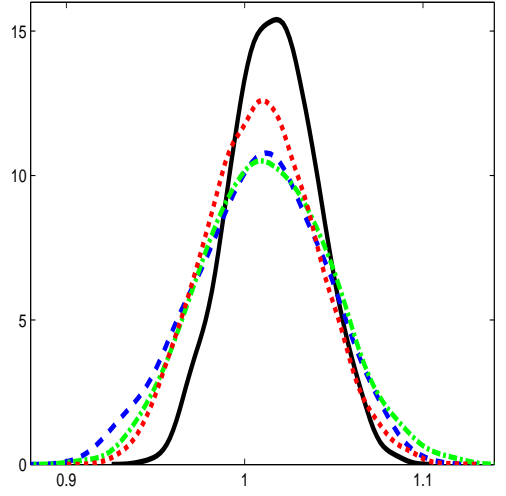

(b) $b$

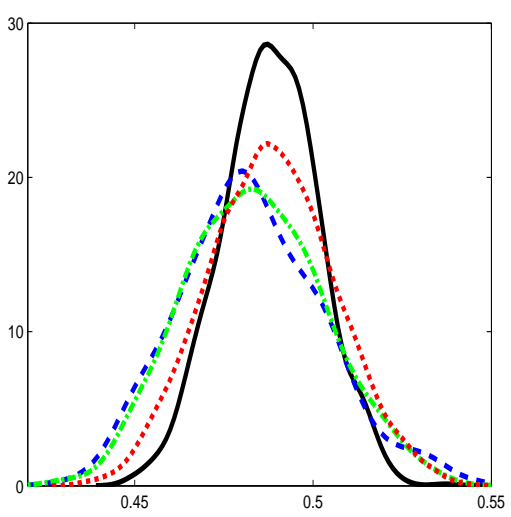

(d) $k$

FIG 3. Posterior distributions for the parameters of the g-and- $k$ model based on the ABC II approaches without applying any regression adjustment. Results are presented for ABC IP (blue dash), $A B C I L$ (green dot-dash), ABC IS (red dot) and from using the true likelihood (black solid). Here a four component normal mixture model was used as the auxiliary model (on-line figure in colour). 
test statistic

$$
T(\phi)=\sum_{i=1}^{N} \frac{\left(m_{i}-\mu_{i}(\phi)\right)^{2}}{V_{i}(\phi)}
$$

where $\mu_{i}(\phi)$ and $V_{i}(\phi)$ denote the mean and variance of the beta-binomial model for observation $i$, respectively, which depend on the auxiliary parameter $\phi$. For the beta-binomial model, these are given by

$$
\begin{aligned}
\mu_{i} & =\frac{l_{i} \alpha_{i}}{\alpha_{i}+\beta_{i}}, \\
V_{i} & =\frac{l_{i} \alpha_{i} \beta_{i}}{\left(\alpha_{i}+\beta_{i}\right)^{2}\left(\alpha_{i}+\beta_{i}+1\right)},
\end{aligned}
$$

where the dependence of $\mu_{i}, V_{i}, \alpha_{i}$ and $\beta_{i}$ on $\phi$ has been dropped for notational convenience. Here we found that $T(\phi(y))=229.1$. Assuming that this test statistic has a chi-square distribution with degrees of freedom $N-5$, the p-value obtained was roughly 0.14 . To avoid making a distributional assumption about the test statistic, we performed a hypothesis test via Monte Carlo simulation where the null hypothesis was that the observed data can be described by a beta-binomial distribution with parameter $\phi(\boldsymbol{y})$. Based on 1000 Monte Carlo draws from the null hypothesis, we found that the simulated test statistic values exceeded that of the observed test statistic about $18 \%$ of the time. Either way, there is insufficient evidence against the assumption that the beta-binomial distribution provides a good description of the data.

Figure 4 shows the results for pdBIL for $n=1$ (100000 iterations), $n=10$ (50000 iterations) and $n=50$ (10000 iterations) using a suitable thinning interval. The acceptance probabilities were roughly $14 \%, 32 \%$ and $39 \%$ for $n=1, n=10$ and $n=50$ respectively. In all cases, about 3 hours of computing time was required. Here we see an increase in precision for $\nu$ and $\mu_{L}$ as $n$ is increased.

Figure 5 includes results for the ABC II methods. ABC IP and ABC IL were run for 1 million iterations and the tolerance was tuned to obtain an acceptance probability of approximately $1.5 \%-2 \%$ (roughly 20 hours of computing each). ABC IS was run for 20 million iterations due to the available analytic score for the auxiliary likelihood (15 hours of computing and an acceptance rate of roughly

imsart-sts ver. 2013/03/06 file: Bayes_Ind_inf_Revision_For_SS_Supplement.tex date: July 15, 2014 


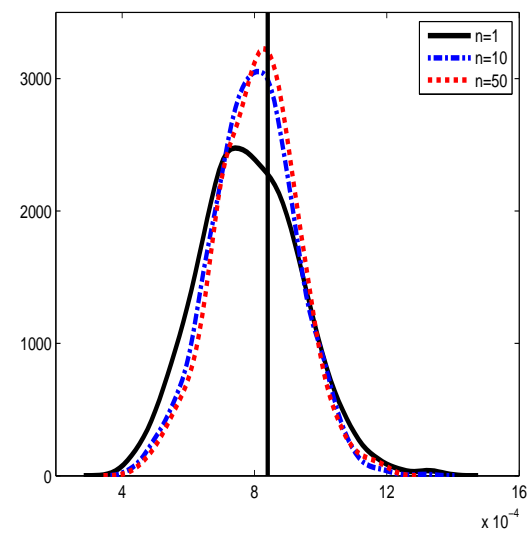

(a) $\nu$

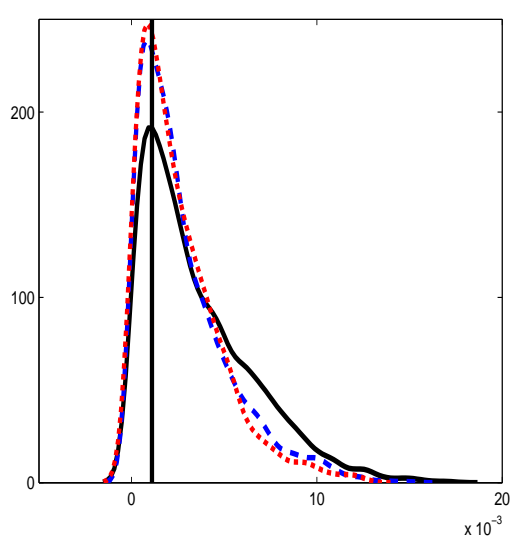

(c) $\mu_{L}$

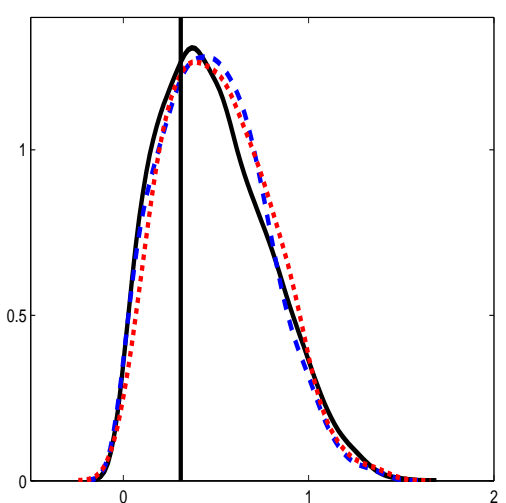

(b) $\mu_{I}$

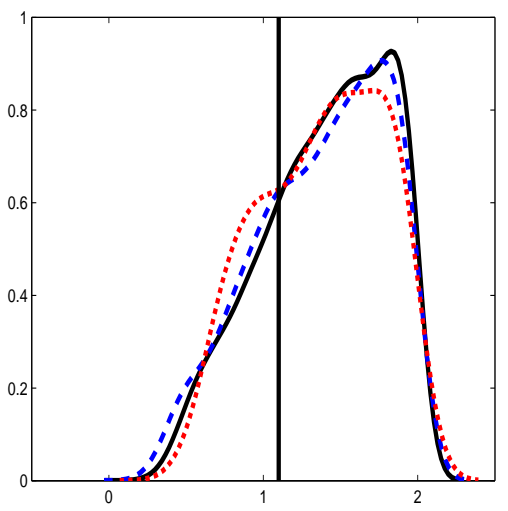

(d) $\beta$

Fig 4. Posterior distributions for the parameters ((a) $\left.\nu,(b) \mu_{I},(c) \mu_{L},(d) \beta\right)$ of the macroparasite model based on an pdBIL approach with $n=1$ (black, solid), $n=10$ (blue, dashed) and $n=50$ (red, dotted) applied to the simulated data (on-line figure in colour). 
$0.2 \%)$. It is evident from the graph that generally the pBII methods are able to recover $\nu$ and $\mu_{L}$ quite precisely, and $\mu_{I}$ but less so. Given that the observed data appear to be quite uninformative about $\mu_{I}$ and $\beta$ (see Drovandi, Pettitt and Faddy (2011)), this simulation study provides a validation of the pBII approaches. Of the pBII methods, the ABC IS method appeared to give the most precise results for $\nu$ and $\mu_{L}$.

It is unclear if the performance of $\mathrm{ABC}$ IS is superior to the other $\mathrm{ABC}$ II methods due to the choices of the ABC tolerance. Here we applied a regression adjustment for the parameters $-\log (\nu)$ and $\sqrt{\mu_{L}}$ in an attempt to reduce the effect of the ABC tolerance. Figure 6 shows the results. We found slightly more precise inferences for the $\mathrm{ABC}$ IP and $\mathrm{ABC}$ IS results and negligible change for ABC IL. Overall, for this example based on this simulated dataset, it appears that ABC IS produced the best results. However, all of the pBII methods performed quite well.

\section{REFERENCES}

Drovandi, C. C. and Pettitt, A. N. (2011). Likelihood-free Bayesian estimation of multivariate quantile distributions. Computational Statistics and Data Analysis 55 2541-2556.

Drovandi, C. C., Pettitt, A. N. and Faddy, M. J. (2011). Approximate Bayesian computation using indirect inference. Journal of the Royal Statistical Society: Series C (Applied Statistics) 60 503-524.

imsart-sts ver. 2013/03/06 file: Bayes_Ind_inf_Revision_For_SS_Supplement.tex date: July 15, 2014 


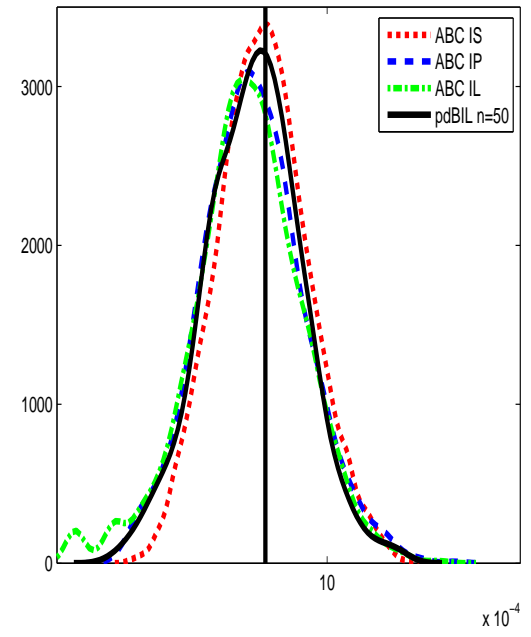

(a) $\nu$

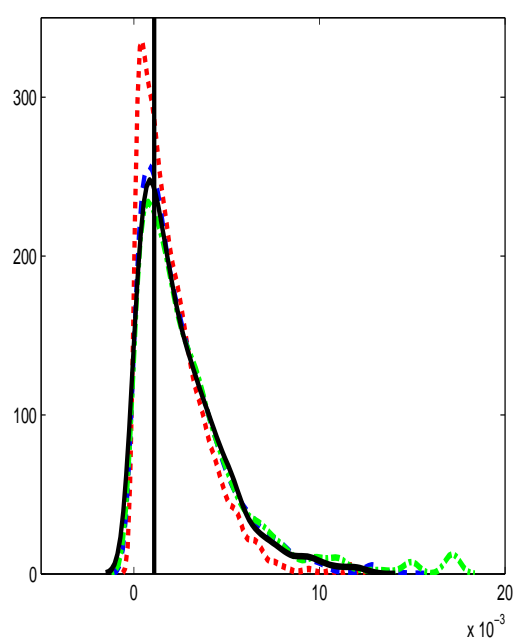

(c) $\mu_{L}$

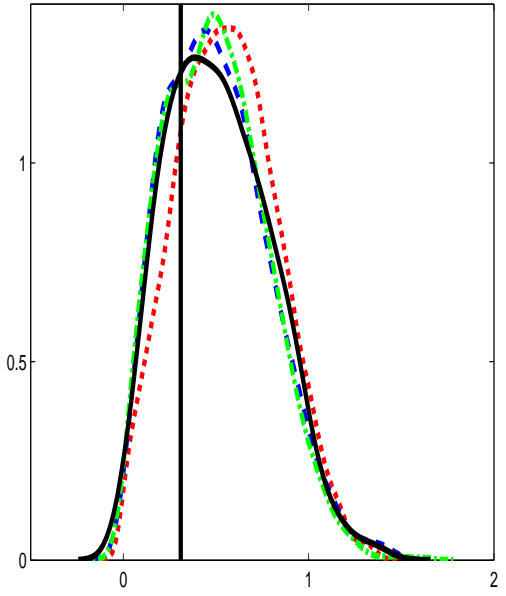

(b) $\mu_{I}$

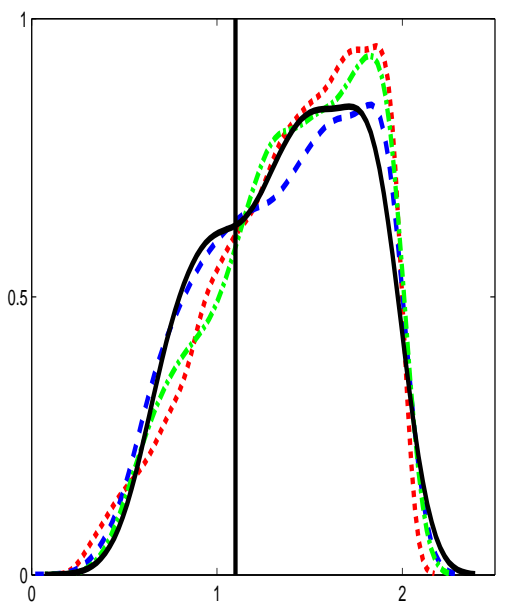

(d) $\beta$

Fig 5. Posterior distributions for the parameters ((a) $\nu$, (b) $\mu_{I}$, (c) $\mu_{L}$, (d) $\beta$ ) of the macroparasite model applied to the simulated data based on ABC IP (blue dash), ABC IS (red dot), ABC IL (green, dot-dash) and pdBIL (black solid) (on-line figure in colour). 


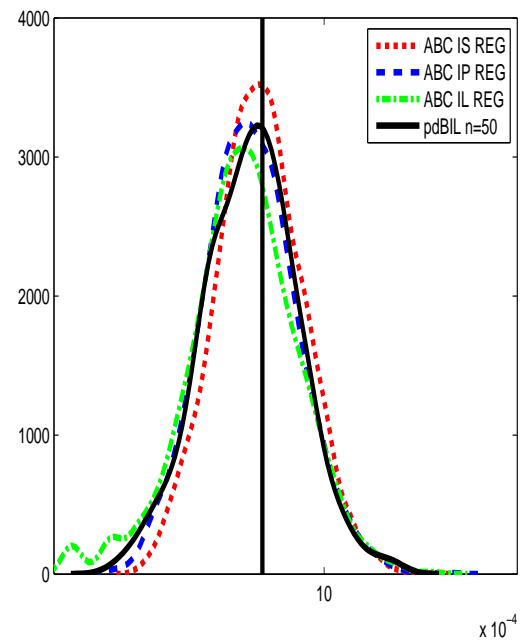

(a) $\nu$

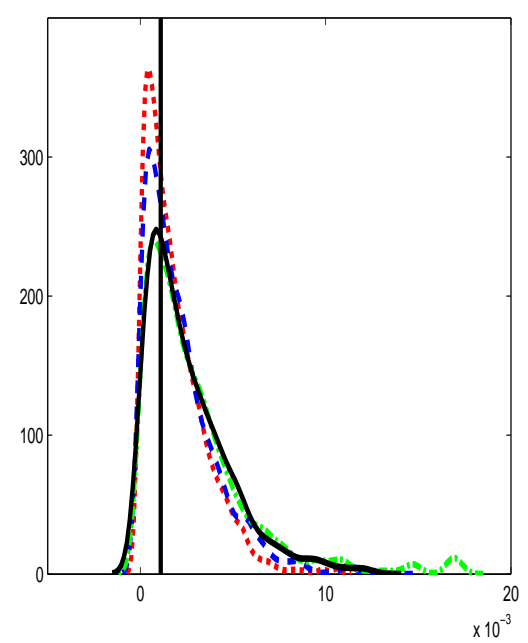

(b) $\mu_{L}$

FIG 6. Posterior distributions for the parameters $\left((a) \nu\right.$ and $\left.(b) \mu_{L}\right)$ of the macroparasite model based on $A B C I P$ (blue dash), ABC IS (red dot), ABC IL (green dot-dash) and pdBIL (black solid) when applied to the simulated data. Regression adjustment has been performed on the output of the ABC II approaches (on-line figure in colour). 